\title{
Group Building Based Power Consumption Scheduling for the Electricity Cost Minimization with Peak Load Reduction
}

\author{
Eunsung Oh *, Jong-Bae Park** and Sung-Yong Son ${ }^{\dagger}$
}

\begin{abstract}
In this paper, we investigate a group building based power consumption scheduling to minimize the electricity cost. We consider the demand shift to reduce the peak load and suggest the compensation function reflecting the relationship between the change of the building demand and the occupants' comfort. Using that, the electricity cost minimization problem satisfied the convexity is formulated, and the optimal power consumption scheduling algorithm is proposed based on the iterative method. Extensive simulations show that the proposed algorithm achieves the group management gain compared to the individual building operation by increasing the degree of freedom for the operation.
\end{abstract}

Keywords: Power consumption scheduling, Group energy management system, Building energy management system, Demand shifting, Peak reduction, Building comfort

\section{Introduction}

With increasing energy conservation in the building sector, there is more attention on the role of buildings with regard to the energy, carbon and environmental footprint. Energy consumption in buildings has been growing in aggregate over time. It has been estimated that $40 \%$ of total energy is consumed by buildings in most International Energy Agency (IEA) member countries, which is more energy consumption than that transportation or industry sectors [1]. Among the main building sectors, more than $60 \%$ of the total energy consumptions are due to the commercial buildings [2].

Energy reduction in buildings can be achieved in many ways from design and construction (i.e., electronic ballasts, LED lighting, Green Roofing, Renewable such as solar panels and photovoltaic cells) to operation. It is reported that, on average, green buildings designed and constructed with an environmental perspective are $28 \%$ more energy efficient than conventional buildings [3]. However, the newly constructed buildings lose $2 \%$ of total floor area [4]. Therefore, while attention to efficiency and other green priorities in new building construction is important in long-term building stock, both short-term and long-term efficiency goals require a focus on efficient operations in existing buildings.

Building Energy Management System (BEMS) is researched as a part of Building Management System (BMS). Conventional BEMSs are forced to fulfill the

$\dagger$ Corresponding Author: Dept. of Electrical Engineering, Gachon University, Korea. (xtra@gachon.ac.kr)

* Dept. of Electrical and Electronic Engineering, Hanseo University, Korea. (esoh@hanseo.ac.kr)

** Dept. of Electrical Engineering, Konkuk University, Korea. (jbaepark @konkuk.ac.kr)

Received: October 21, 2013; Accepted: June 10, 2014 occupants' comfort and by controlling its facilities on Heating, Ventilating, and Air-conditioning (HVAC). Methods for HVAC control, such as rule set based intelligent decision have been proposed $[5,6]$. For the optimal control of specific systems, various fuzzy control and neural networks have been studied [7, 8]. Nevertheless, the conventional BEMS is reactive because they are equipped with simple controllers that track the set-points dictated by the operator. Increasing smart facilities which have more allowable room to control and improving information infrastructure, more proactive control for building energy management is required [9].

In our work, we focus on power consumption scheduling to minimize the electricity cost with peak load reduction in group building environments. A building group means coordinated multiple buildings on large sites such as federal facilities, university and corporate campuses. Differently from controlling individual buildings, integrated operations are performed considering each building's characteristics in the group. Since each building has its own response characteristics, its reaction and corresponding cost is different from other buildings. When imposing a same restriction condition to multiple buildings, it could be too easy for some of them to achieve. On the other hand, it could be too difficult for some others to meet the goal. It would cause higher cost in result. Individual building based approaches have fundamental limitations caused by the difference of a problem space. BEMS based on building group (G-BEMS) could reduce energy losses and to increase the operational opportunities [9]. For peak reduction, demand shifting based on power consumption scheduling is taken into account for G-BEMS. There are some loads which allow the system to shift its demand earlier or late (e.g., pre/post-cooling) [4]. This demand shifting will flatten the aggregated load profile and hence 
reduce the overall cost of purchasing electrical energy. The amount of the peak reduction is assumed to be determined from the historical peak value of individual buildings. We also consider the effect of the building comfort change by demand shifting. The proposed power consumption scheduling minimizes the electricity cost balancing the conflict between energy consumption and building comfort.

We firstly explain a group building energy system model, and discuss the effect of the demand shifting and building comfort change on the cost. Based on that, the power consumption scheduling is formulated as a convex optimization problem. The solution of the problem could reflect the characteristics of buildings and the requirement of overall peak load reduction. Numerical results verify that the power consumption scheduling with G-BEMS saves the cost comparing with individual operations.

The remainder of this paper is organized as follows. In Section 2, we formally describe our system model. The problem formulation and operational algorithm for power consumption scheduling are presented in Section 3. In Section 4, we demonstrate the performance of the proposed power consumption scheduling under group operation environments with two buildings. Finally, we conclude the paper in Section 5.

\section{System Model}

In most of conventional BEMSs, the key functionality is on interactions between the electricity market and each building operator [9]. Under this paradigm, each building communicates with the market individually as depicted in Fig. 1(a). Instead, rather than focusing only on how each building behaves individually, G-BEMS should have the objective that the aggregate demand satisfies some desired properties on a large site with multiple buildings, as depicted in Fig. 1(b).

\subsection{Energy system model}

We consider $T$ (i.e., $\{1, ;|T|\}$ ) observation time. Without loss of generality, we assume that unit time duration is one hour and $|T|=24$.

Consider a group building energy system with a building set $B$ (i.e., $\{1, ;|\cdot B|\}$ ), where the number of buildings is $|B|$. A building set could be constructed from virtual networks using information infrastructure (e.g., franchised retail stores), as well as physical neighboring buildings. A G-BEMS which is connected to electricity markets manages a building group, as shown in Fig. 1(b).

Electricity markets announce the electricity price, $c^{t}$ $[\mathrm{KRW} / \mathrm{kWh}]^{1}$, and the demand $d_{b}^{t}[\mathrm{~kW}]$ is requested for each building $b \in B$ at time $t \in T$. We assume that the electricity price and the required demand could be

${ }^{1} \mathrm{KRW}$ is the currency of South Korea.

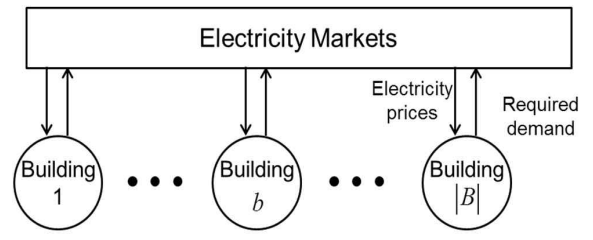

(a) An individual building energy system

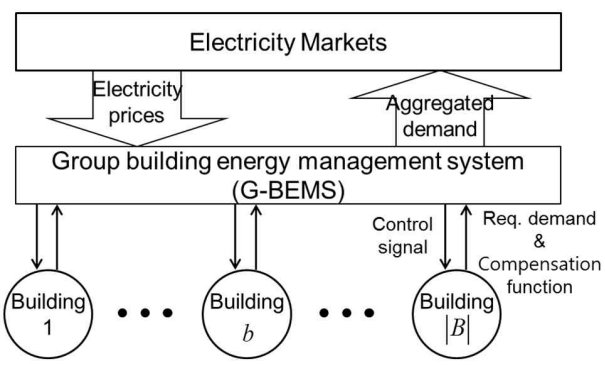

(b) A group building energy system

Fig. 1. Building energy system models

estimated during the observation time ${ }^{2}$.

\subsection{Building model}

The goal of BEMS is not only to manage the energy consumption but also to support the occupants' comfort. When the demand is changed from the required demand $d_{b}^{t}$ owing to the power consumption scheduling, the building operator should compensate the occupants for increasing inconvenience.

The definition of comfort level for reflecting the occupants' comfort is a complex and challenging problem because there are many factors, but the relation between the comfort level and the electricity usage is mathematically expressed as a quadratic function in building operation $[12,13]$. Therefore, the compensation expenses to the occupants could be represented as a quadratic function by the amount of demand change, $\Delta d_{b}^{t}$, as follow:

$$
u_{b}^{t}\left(\Delta d_{b}^{t}\right)=\alpha_{b} \cdot\left(\beta_{b}^{t} \cdot \Delta d_{b}^{t}\right)^{2}[\mathrm{KRW}]
$$

where $\alpha_{b}$ is a scale factor determined by the building type and characteristic such as office, hospital and university campus, and $\beta_{b}^{t}$ means the weighting factor reflected the demand characteristic of building $b$ at time $t$.

For an example, when a compensation function is modeled as

$$
u_{b}^{t}\left(\Delta d_{b}^{t}\right)=\alpha_{b} \cdot\left(\Delta d_{b}^{t} / d_{b}^{t}\right)^{2}
$$

\footnotetext{
2 There are many studies (e.g., historical data set matching and customer base line modeling) which could pre-determine the electricity price and the demand within $1-2 \%$ estimation error $[10,11]$. But, to focus on the effect of the group management, we consider the perfect estimation case.
} 


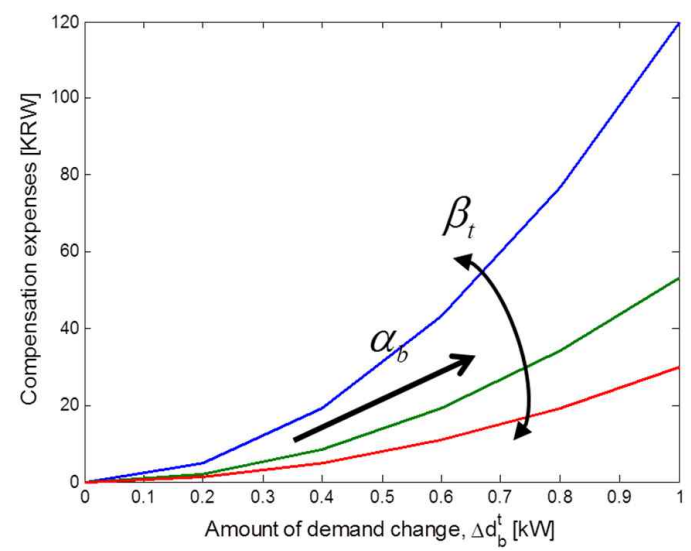

Fig. 2. An example of a compensation function by factors

and $\alpha_{b}=120, d_{b}^{t}=1,1.5,2$, it is illustrated in Fig. 2 . As shown in Fig. 2, the compensation factor $\alpha_{b}$ decides the main slope of compensation function during the observation time. When the occupants are sensitive to the energy consumption, $\alpha_{b}$ has large value. The compensation factor $\beta_{b}^{t}$ changes around the main slope, and reflects the condition of the required demand at each time $t$. In (2), more compensation expense is needed when the required demand is small because there is less room to control.

\section{Power Consumption Scheduling for G-BEMS}

\subsection{Problem formulation}

In this paper, we aim at proposing a power consumption scheduling algorithm for G-BEMS that minimizes the electricity cost with peak load reduction.

The electricity cost is calculated as

$$
O\left(c^{t}, d_{b}^{t}, \Delta d_{b}^{t}\right)=\sum_{t \in T} \sum_{b \in B}\left\{c^{t} \cdot\left(d_{b}^{t}-\Delta d_{b}^{t}\right)+u_{b}^{t}\left(\Delta d_{b}^{t}\right)\right\}
$$

The first term is the electricity bill, and the second term is the compensation expenses.

Because the electricity price $c^{t}$ and the required demand $d_{b}^{t}$ are the observed values, the object function could be written as

$$
O\left(\Delta d_{b}^{t}\right)=\sum_{t \in T} \sum_{b \in B}\left\{-c^{t} \cdot \Delta d_{b}^{t}+u_{b}^{t}\left(\Delta d_{b}^{t}\right)\right\}
$$

Even if the demand is shifted to the pre-/post-time slot from the required time, the required demand should be supported. Constraints for the required demand of each building are added,

$$
\sum_{t \in T}\left(d_{b}^{t}-\Delta d_{b}^{t}\right)-\sum_{t \in T} d_{b}^{t} \geq 0 \rightarrow \sum_{t \in T} \Delta d_{b}^{t} \leq 0, \forall b \in B
$$

From the purpose of the our power consumption scheduling, the second constraint expresses the condition about the peak load limitation,

$$
\sum_{b \in B}\left(d_{b}^{t}-\Delta d_{b}^{t}\right) \leq d^{*}, t \in T
$$

where $d^{*}$ is the maximum peak load threshold.

In general, our power consumption scheduling problem is formulated as

$$
\begin{array}{ll}
\min _{\Delta d_{b}^{t}} & O\left(\Delta d_{b}^{t}\right) \\
\text { s.t. } & \sum_{t \in T} \Delta d_{b}^{t} \leq 0, \forall b \in B, \\
& \sum_{b \in B}\left(d_{b}^{t}-\Delta d_{b}^{t}\right) \leq d^{*}, \forall t \in T .
\end{array}
$$

\subsection{Proposed power consumption scheduling algorithm}

The problem to minimize the electricity cost formulated in (7) is the convex optimization problem because the constraints are satisfied the convexity and the object function is convex [14]. Therefore, the problem could be solved the Lagrangian relaxation method without loss of the optimality.

Using the Lagrangian multiplier $\lambda_{b}$ and $v_{t}$, we can relax the constraints of the problem (7), as follow:

$$
\begin{aligned}
L= & \sum_{t \in T} \sum_{b \in B}\left\{-c^{t} \cdot \Delta d_{b}^{t}+u_{b}^{t}\left(\Delta d_{b}^{t}\right)\right\}+\sum_{b \in \mathcal{B}} \lambda_{b} \sum_{t \in T} \Delta d_{b}^{t} \\
& +\sum_{t \in T} v_{t} \sum_{b \in \mathcal{B}}\left(d_{b}^{t}-\Delta d_{b}^{t}-d^{*} /|\mathcal{B}|\right) .
\end{aligned}
$$

From Karush-Kuhn-Tucker (KKT) and slackness conditions, the condition should be satisfied,

$$
\begin{aligned}
\lambda_{b} \cdot \sum_{t \in T} \Delta d_{b}^{t} & =0, \quad \forall b \in \mathcal{B}, \\
v_{t} \cdot \sum_{b \in \mathcal{B}}\left(d_{b}^{t}-\Delta d_{b}^{t}-d^{*} /|\mathcal{B}|\right) & =0, \quad \forall t \in T, \\
\nabla u_{b}^{t}\left(\Delta d_{b}^{t}\right)+\left(\lambda_{b}-v_{t}-c^{t}\right) & =0, \quad \forall b \in \mathcal{B}, t \in T, \\
\lambda_{b}, v_{t} & \geq 0, \quad \forall b \in \mathcal{B}, t \in T .
\end{aligned}
$$

We now solve the above equation. First, check the Lagrangian condition for each building from the first condition. The Lagrangian multipliers have zero or some positive value. For the building $b$, when $\lambda_{b}$ becomes zero, the first constraint of (7) cannot be satisfied because the amount of demand shifting is always positive in (10), (i.e., $\left.c^{t}, v_{t} \geq 0\right)$. Therefore, from the first condition of (9), it is determined

$$
\lambda_{b}>0 \text { and } \sum_{t \in T} \Delta d_{b}^{t}=0
$$

It means that the total amount of demand shifting at each 
building must be zero to minimize the operational cost.

In a similar manner, for each observation time $t$, the Lagrangian multiplier $v_{t}$ is calculated from the second condition of (7),

$$
v_{t}=\left\{\begin{array}{cc}
0, & \text { if } \sum_{b \in \mathcal{B}}\left(d_{b}^{t}-\Delta d_{b}^{t}\right) \neq d^{*}, \\
\text { some value, } & \text { if } \sum_{b \in \mathcal{B}}\left(d_{b}^{t}-\Delta d_{b}^{t}\right)=d^{*} .
\end{array}\right.
$$

Finally, using the compensation expenses function in (2) and the third condition of (9), the optimum amount of demand shifting is calculated as,

$$
\Delta d_{b}^{t}=\frac{c^{t}-\lambda_{b}+v_{t}}{2 \cdot \alpha_{b} \cdot\left(\beta_{b}^{t}\right)^{2}}, \forall b \in \mathcal{B}, t \in T
$$

To determine the amount of demand shifting, G-BEMS is to update the Lagrangian multiplier $\lambda_{b}$ and $v_{t}$ using a subgradient method in the range of (10) and (11) [14].

The proposed power consumption scheduling algorithm for G-BEMS is summarized as follows:

\section{[The optimal power consumption scheduling algorithm]}

Step 0) Estimate $d_{b}^{t}$ and $c^{t}$ in $b \in \mathcal{B}$ and $t \in T$.

Step 1) Update the Lagrangian multiplier $\lambda_{b}$ and $v_{t}$, as follow:

1) Initialize $\lambda_{b}^{(0)}, v_{t}^{(0)}$ and $\varepsilon$.

2) Given $\lambda_{b}^{(l)}$ and $v_{t}^{(l)}$, calculate the Eq. (12).

3) Perform a subgradient update:

$$
\begin{aligned}
& \lambda_{b}^{(l+1)}=\left[\lambda_{b}^{(l)}+l^{(l)} \cdot \sum_{t \in T} \Delta d_{b}^{t}\right]^{+}, \forall b \in \mathcal{B}, \\
& v_{t}^{(l+1)}=\left[v_{t}^{(l)}+\kappa^{(l)} \cdot\left(\sum_{b \in \mathcal{B}}\left(d_{b}^{t}-\Delta d_{b}^{t}\right)-d^{*}\right)\right]^{+}, \forall t \in T
\end{aligned}
$$

where $[x]^{+}=\max \{0, x\}$.

4) Return to 2) until $\left|\lambda_{b}^{(l+1)}-\lambda_{b}^{(l)}\right|<\varepsilon$ and

$$
\left|v_{t}^{(l+1)}-v_{t}^{(l)}\right|<\varepsilon \text {. }
$$

where $\varepsilon$ is a stopping criterion which is chosen as a very small value.

Step 2) Determine the amount of demand shifting, $\Delta d_{b}^{t}$, substituting the converged $\lambda_{b}$ and $v_{t}$ into (12)

The subgradient method is guaranteed to converge on the optimal variable for the convex problem if the step sizes $t^{(l)}$ and $\kappa^{(l)}$ are chosen using a diminishing step size rule [14].

\section{Numerical Results}

For our simulation, we consider a building group with two buildings which have compensation functions as

$$
u_{b}^{t}\left(\Delta d_{b}^{t}\right)=\alpha_{b} \cdot\left(\Delta d_{b}^{t} / d_{b}^{t}\right)^{2}, \forall b \in \mathcal{B}=\{1,2\}
$$

We assume that the electricity price is constant as $c^{t}=C=120 \quad[\mathrm{KRW} / \mathrm{kWh}]$ during the observation time which is a typical value of Korea grid model [15], and the total required demand of buildings is

$$
\sum_{b \in B} d_{b}^{t}=5+3 \cdot \sin (2 \pi \cdot(t-6) / T),
$$

which has $8 \mathrm{~kW}$ peak demand and $120 \mathrm{kWh}$ total energy consumption during one day. The required demand of each building is modeled as $d_{1}^{t}=x \cdot d^{t}$ and $d_{2}^{t}=(1-x) \cdot d^{t}$ where $x$ (i.e., $0 \leq x \leq 1)$ is the required demand ratio.

We compare the performance of the proposed power consumption scheduling for building group to that of the individual building operation which has the objective function,

$$
O_{b}\left(\Delta d_{b}^{t}\right)=\sum_{t \in T}\left\{-c^{t} \cdot \Delta d_{b}^{t}+u_{b}^{t}\left(\Delta d_{b}^{t}\right)\right\}
$$

and constraints.

\subsection{Case studies}

Fig. 3 shows an example of group and individual power consumption scheduling results when $x=0.7$ and $5 \%$ peak load reduction (i.e., $d^{*}=7.6 \mathrm{~kW}$ for group operation, and $d_{1}^{*}=5.32 \mathrm{~kW}$ and $d_{2}^{*}=2.28 \mathrm{~kW}$ for individual operation). Solid lines without mark, blue solid lines with circle and red dashed lines with square express the original required demand, the results of group and individual operation, respectively.

In the upper figure of Fig. 3, the demand of the first building is more shifted by the group operator than that by the individual operator at peak time, and vice versa in the lower figure of Fig. 3. This is because the compensation
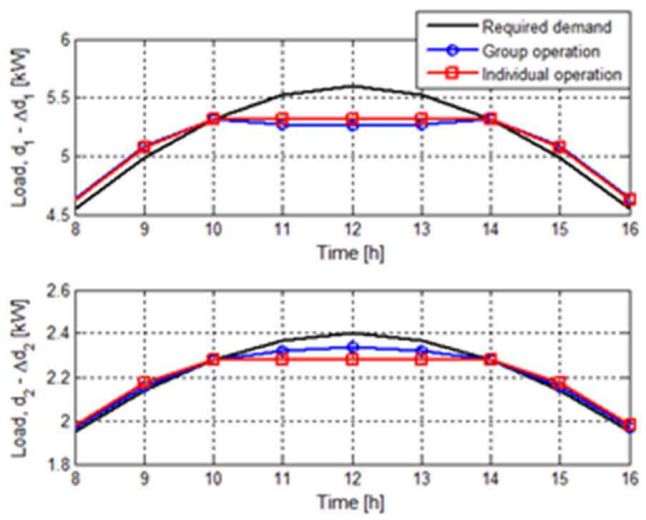

Fig. 3. Comparison of results between group and individual power consumption scheduling for building 1 and 2 


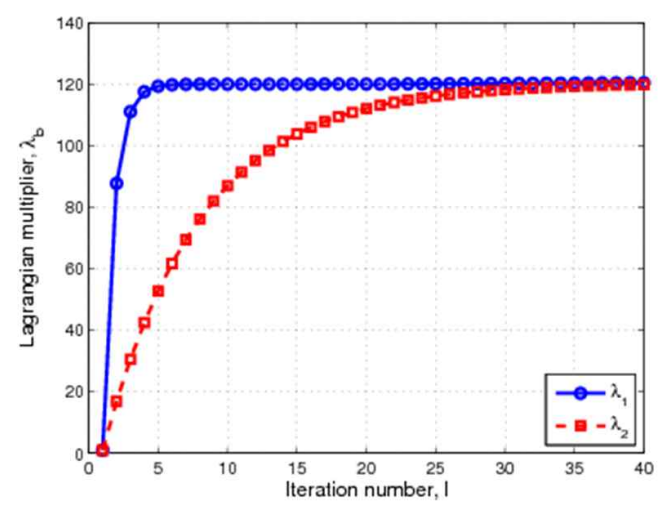

Fig. 4. Convergence of the Lagrangian multiplier, $\lambda_{b}$

expenses of the second building have higher prices than that of the first building. To satisfy the peak load reduction constraint, the individual operation should shift its own load from peak time, but the group operation can adjust the amount of shifting load considering the compensation function of each building. The group management could be achieved the operation gain as increasing the degree of freedom for the operation.

The proposed power consumption scheduling based on Lagrangian relaxation is solved by the iterative method. The iteration process to achieve the results in Fig. 3 is illustrated in Fig. 4. Even though the number of iteration is different related to the amount of the shifted demand, the solution is always converged to the global optimum because the original problem satisfies the convexity [14].

\subsection{Characteristics: Demand ratio and compensation factor}

The operation gain of the group management is related to the degree of freedom for the operation, which depends on the required demand and the compensation function. Figs. 5 and 6 show the group management gain through the demand ratio, $x$, and compensation factor, $\alpha_{b}$. The group management gain is defined as

$$
\operatorname{Gain}[\%]=100 \times \frac{\sum_{b \in B} O_{b}\left(\Delta d_{b}^{t}\right)-O\left(\Delta d_{b}^{t}\right)}{\sum_{b \in B} O_{b}\left(\Delta d_{b}^{t}\right)} .
$$

Fig. 5 illustrates the group management gain when the required demand ratio, $x$, is changed from 0.1 to 0.9 with $\alpha_{1}=C, 2 C, 3 C$ and $\alpha_{2}=C$. The group operation cannot achieve any gain when the required demand and the compensation function of each building are same (e.g., $x=0$ and $\alpha_{1}=\alpha_{2}=C$ ). When the asymmetry of the required demands between buildings is increasing, the group management gain is improved. It is because the group operator could have more room to control for satisfying the constraints when the asymmetry of building characteristics between buildings is increasing. The group

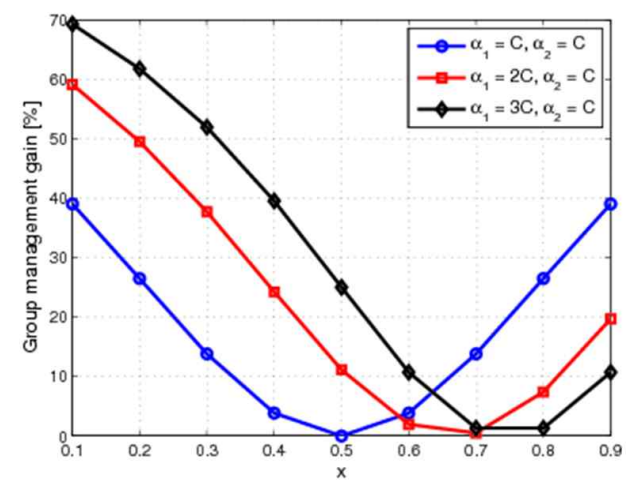

Fig. 5. Group management gain versus the required demand ratio, $x$

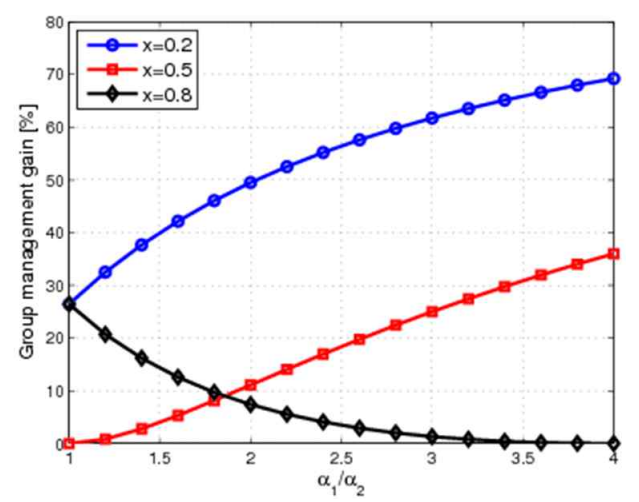

Fig. 6. Group management gain versus the compensation factor, $\alpha_{b}$

management gain is obviously maximized when the building, which has larger room (larger required demand) to control, requires lower compensation expenses such as $x=0.1, \alpha_{1}=3 C$, and $\alpha_{2}=C$.

The relationship between the group management gain and the compensation expenses is presented in Fig. 6 . When the required demand ratio is less than 0.5 , the group management gain is enhanced with increasing the ratio of the compensation factor. However, the opposite trend is shown when the required demand ratio is exceeding 0.5 . This is because the degree of freedom for the operation is reduced by going up the compensation expenses. It is said that the group management gain could be achieved by the asymmetric characteristics between buildings, but, to enhance the group management gain (to minimize the cost), we should compose a building group to maximize the degree of freedom for the operation.

Fig. 7 shows the group management gain considering three buildings with $\alpha_{1}=\alpha_{2}=\alpha_{3}=C$. The required demand of each building is modeled using the demand ratio $x$ and $y$ such as $d_{1}^{t}=x \cdot d^{t}, d_{2}^{t}=y \cdot d^{t}$ and $d_{3}^{t}=$ $(1-x-y) \cdot d^{t}$ where $x \in\{0.1,0.3\}$ and $0.1 \leq y \leq 0.9-x$. Similar to the result of two buildings, increasing the asymmetry of the required demands among buildings, the more group management gain is achieved. In addition, comparing the group management gain when the required 


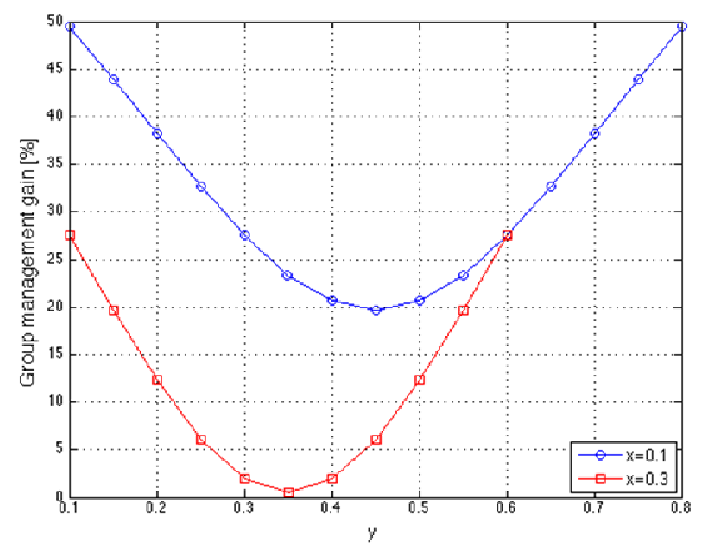

Fig. 7. Group management gain versus required demand ratio, $x$ and $y$ considering three buildings

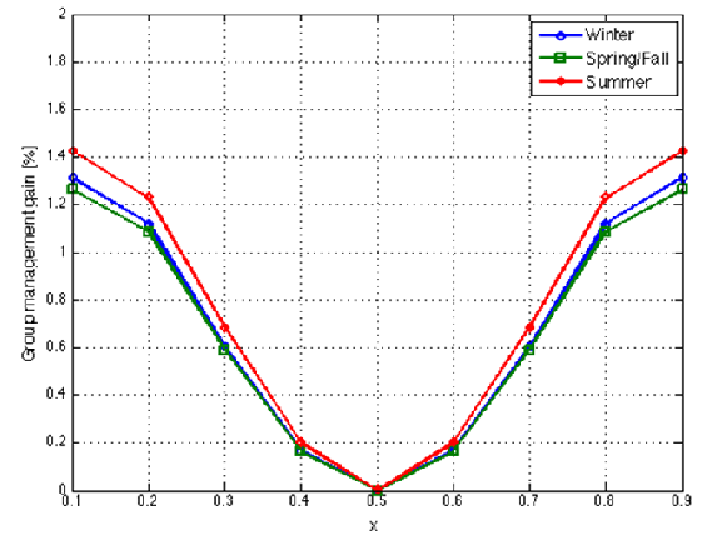

Fig. 8. Group management gain versus the required demand ratio, $x$ in TOU pricing

demand ratio is $(0.1,0.9)$ for two buildings and $(0.1,0.1$, 0.8 ) for three buildings, it could be said that the group management gain will be improved with increasing number of buildings.

In Fig. 8, the effect of dynamic pricing versus the change of the required demand ratio is presented using time of unit pricing of KEPCO Industrial (A)-II tariff [16]. The group management gain is enhanced when the asymmetry of the required demand and the variance of pricing are increasing. However, the group management gain has a small value comparing to that with the constant pricing. This is because the electricity cost is affected by the peak shifting rather than the group management. Even if the reduction of electricity cost is related with many characteristics such as tariff, compensation function and peak shifting policy, the group management is additionally enhancing the room to reduce the electricity cost.

\subsection{Characteristics: Time offset between the required demands}

The operation is affected by the temporal characteristic, as well as the building characteristic. We investigate the effect of the time offset, which is the difference between

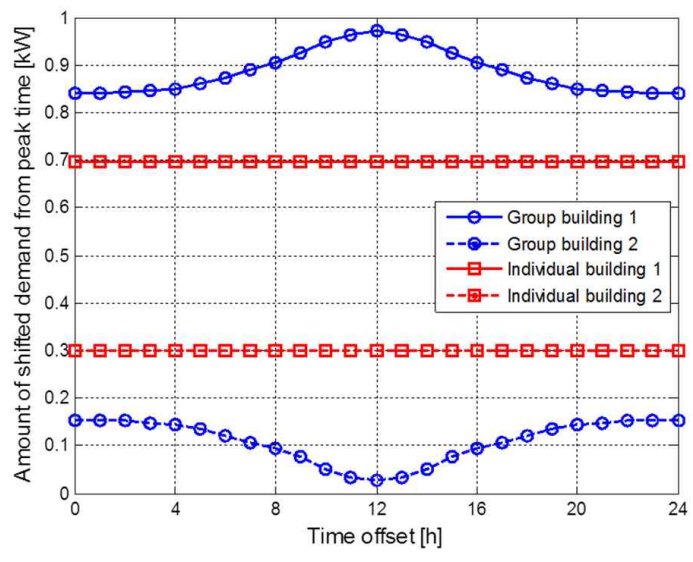

Fig. 9. Comparison of the amount of shifted demand from peak time between group and individual operations

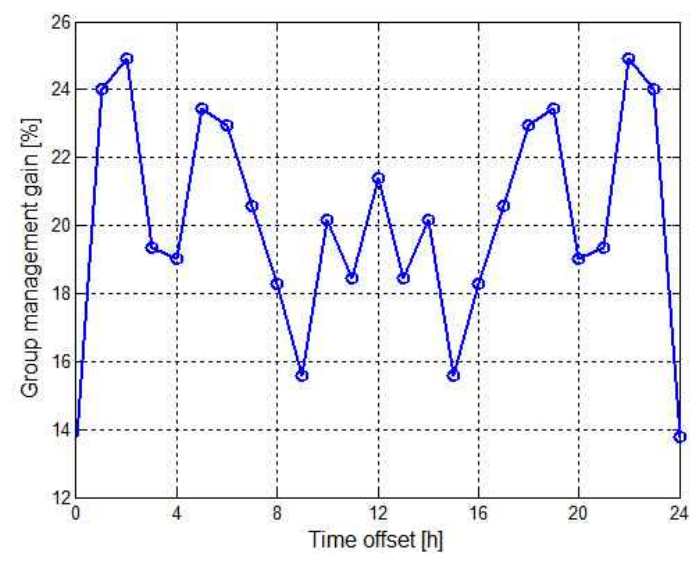

Fig. 10. Group management gain versus time offset between the required demands

the peak times of each building's required demand, with $\alpha_{1}=\alpha_{2}=C$ and $5 \%$ peak load reduction. The required demand is assumed

$$
\begin{aligned}
& d_{1}^{t}=0.7 \times\{5+3 \cdot \sin (2 \pi \cdot(t-6) / T)\} \\
& d_{2}^{t}=0.3 \times\left\{5+3 \cdot \sin \left(2 \pi \cdot\left(t-6-T_{\text {offset }}\right) / T\right)\right\} .
\end{aligned}
$$

Fig. 9 shows how much demand is shifted from the peak time. As discussed above, the amount of shifted demand from peak time is always same by the individual operation because it is considering its own demand only.

However, with increasing the time offset (increasing the time between the peak demands of each building), the group operator is more shifting the demand of the building which has the highest peak demand. It is said that the group operation could autonomously flatten the aggregated load profile considering the temporal characteristic of the required demand.

In Fig. 10, the group management gain is presented versus the time offset. The group management gain doesn't become a monotonic function because the compensation expense has not linearity on the amount of the shifted 
demand. However, when the required demands between buildings have the time offset (the asymmetry of the temporal characteristic), the group management gain is enhanced comparing to that without the time offset.

\section{Conclusion}

In this paper, we focused on the problem of power consumption scheduling for G-BEMS to minimize the electricity cost in group building environments. In particular, we considered the demand shifting to meet the given amount of peak load reduction. Taking into account the relationship between the building energy consumption and the occupants' comfort, we proposed the power consumption scheduling algorithm. Simulation results verified that the proposed algorithm achieves the gain compared to the results of the individual operation. In addition, we observed the correlation between the group management gain and the building characteristics such as the required demand and the compensation expenses, and suggested the guide to construct the energy effective building group which is an open problem for future study.

\section{Acknowledgements}

This work was supported by the 'Energy Efficiency \& Resources' of the Korea Institute of Energy Technology Evaluation Panning(KETEP) grant funded by the Korea government Ministry of Trade, Industry and Energy (No. 20119010200010).

\section{References}

[1] "Energy efficiency requirements in building codes, energy efficiency policies for new buildings," White Paper, Int. Energy Agency (IEA), Mar. 2008

[2] "Buildings energy data book," White Paper, U.S. Dept. of Energy, Mar. 2012

[3] "The Costs and Benefits of Green Buildings", A Report to California's Sustainable Building Task Force, Oct. 2003

[4] "Energy efficiency trends in residential and comercial buildings," White Paper, U.S. Dept. of Energy, Aug. 2010

[5] H. Doukas, K. D. Patlitzianas, K. Iatropoulos, and J. Psarras, "Intelligent building energy management system using rule sets," Building and Environment, vol. 42, No. 10, pp. 3562-3569, Oct. 2007

[6] J. Han, Y. K. Jeong, and I. Lee, "Efficient Building Energy Management System Based on Ontology, Inference Rules, and Simulation," in Proc. of 2011 IACSIT Singapore Conference, vol. 5, Sep. 2011, pp.
295-299

[7] R. Alcala, J. Casillas, O. Cordon, A. Gonzalez, and F. Herrera "A genetic rule weighting and selection process for fuzzy control of heating, ventilating and air conditioning systems," Artificial Intelligence, Vol. 18, No. 3, pp. 279-296, Apr. 2005

[8] A. I. Dounis and C. Caraiscos, "Advanced control systems engineering for energy and comfort management in a building environment - A review," Renewable and Sustainable Energy Reviews, vol. 13, no. 6, pp. 1246-1261, Aug. 2009

[9] V. M. Zavala, C. Thomas, M. Zimmerman, and A. Ott, "Next-generation building energy management systems and implications for electricity markets," Tech. Paper (ANL/MCS-TM-315), Argonne National Lab., Jul. 2011

[10] Young-Min Wi, Ji-Hui Kim, Sung-Kwan Joo, JongBae Park, and Jae-Chul Oh, "Customer baseline load (CBL) calculation using exponential smoothing model with weather adjustment," in Proc. of IEEE T\&D Conf. \& Expo.: Asia and Pacific, Mar. 2009, pp. $1-4$

[11] Almas Heshmati, "Survey of models on demand, customer base-line and demand response and their relationships in the power market," IZA Discussion Paper, no. 6637, Jun. 2012

[12] R. Yang and L. Wang, "Multi-objective optimization for decision-making of energy and comfort management in building automation and control," Sustainable Cities and Society, vol. 2, no. 1, pp. 1-7, Feb. 2012

[13] H. Park, N. Martaj, M. Ruellan, R. Bennacer, and E. Monmasson, "Modeling of a Building System and its Parameter Identification," J. Electr. Eng. Technol., vol. 8, no. 5, pp. 975-983, Sep. 2013

[14] S. Boyd and L. Vandenberghe, Convex Optimization. Cambridge, CB2 2RU, UK: Cambridge University Press, 2004.

[15] Statistics of electric power in Korea, White Paper, Korea Electric Power Corporation, Jun. 2012.

[16] Electric rate table, KEPCO, Nov. 2013.

[17] E. Oh, S.-Y. Son, and J.-B. Park, "An Optimal Power Consumption Scheduling considering Comfort Level based on the Group Energy Management Scheme," in Proc. of ISGC\&E 2013, Jul. 2013, pp. 746-750.

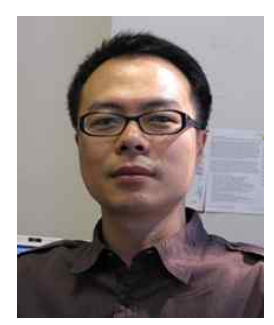

Eunsung Oh $\mathrm{He}$ received his B.S., M.S. and Ph.D. degrees in Electrical Engineering at Yonsei University, Seoul, Korea, in 2003, 2006 and 2009, respectively. From 2009 to 2011, he was a post-doctoral researcher in the Department of Electrical Engineering at the University of Southern California's Viterbi School of Engineering. From 2011 to 2012, 
he was a senior researcher at Korea Institute of Energy Technology Evaluation and Planning, Korea. From 2012 to 2013, he was a research professor in the Department of Electrical Engineering at Konkuk University, Korea. He is currently an assistant professor in the Department of Electrical and Computer Engineering at Hanseo University, Korea. His main research interests include the design and analysis of algorithms for green communication networks and smart grid.

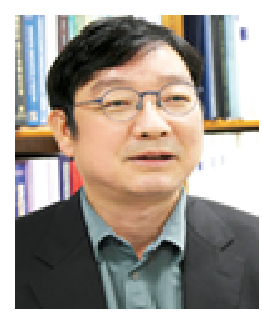

Jong-Bae Park He received his B.S., M.S. and Ph.D. degrees in Electrical Engineering at Seoul National University Seoul, Korea, in 1987, 1989 and 1998, respectively. He is currently a professor in the Dept. of Electrical Engineering at Konkuk University, Korea. He was a researcher of Korea Electric Power Corp. (KEPCO) during 1989-1998 and a resident researcher of Electric Power Research Institute (EPRI) during 2006-2008, respectively. His main research interests include the power system economics, operations and planning.

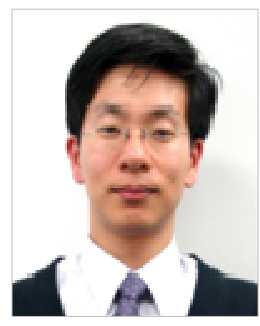

Sung-Yong Son He received the B.S. and M.S. degrees from Korea Advanced Institute of Science and Technology (KAIST), Korea in 1990 and 1992, respectively, and $\mathrm{Ph} . \mathrm{D}$. degree in Mechanical Engineering from University of Michigan, Ann Arbor, in 2000. From 2000 to 2005, he worked at 4DHomeNet and Icross-technology, respectively. He is an associate professor in the Department of Electrical Engineering, Gachon University, Korea. His main research interests include smart grid and smart home. 